\title{
Primary surgery effect on dental arch relationships of patients born with unilateral cleft lip and palate using the GOSLON yardstick index
}

SADJ June 2021, Vol. 76 No. 5 p245 - p249

E Ghabrial', HJS van den Berg ${ }^{2}$, SM du Plessis ${ }^{3}$

\section{ABSTRACT}

\section{Objectives}

To score dental arch development using the Great Ormond Street, London, and Oslo (GOSLON) yardstick index, following primary surgery in patients with a complete unilateral cleft lip and palate (UCLP), and to compare the outcome score with the GOSLON score of Cleft Care UK (CCUK) as well as with the Clinical Standards Advisory Group (CSAG), United Kingdom.

\section{Methods}

Study models of patients (average age 12 years) with a non-syndromic complete UCLP, who had been surgically treated at the University of Pretoria Facial Cleft Deformity (UPFCD) clinic. They were assessed using the GOSLON yardstick index by certified raters from the Dental School in Dundee, Scotland. The mean outcome ratings were calculated from the scoring of 27 sets of plaster models. The other scoring rounds were used to calculate intraand inter-observer agreement using Cohen's weighted kappa and Fleiss's multi-rater kappa.

\section{Results}

There were strong intra- and inter-observer agreement, with a weighted kappa of 0.92. The Facial Cleft Deformity (FCD) clinic data showed a good treatment outcome with a mean GOSLON rating of 2.85 compared to a rating of 3.2 for the CSAG and 2.62 for the CCUK cohort studies.

\section{Author affiliations:}

1. Emad Ghabrial: $B c h D, M c h D$ (Orthod), $P h D$, Senior Lecturer University of Pretoria, Pretoria, South Africa.

ORCID Number: 0000-0002-7213-9760

2. Hester JS van den Berg: Dip/GenN, Dip/CommNSc, Dip/PsychN, Gauteng Health, Community health nurse in Maxillofacial oral surgery department, University of Pretoria, Pretoria, South Africa.

3. Susanna M du Plessis: Dip/GenN, Dip/CommNsc, Dip/NAdmin, Community health nurse, Pretoria, South Africa

Corresponding author: Emad Ghabrial

Faculty of Health Sciences, Department of Orthodontics, University of

Pretoria, PO Box 1266, Pretoria 0001, South Africa.

Email: emad.ghabrial@up.ac.za

Author contributions:

1. Emad Ghabrial: Primary author - $80 \%$

2. Hester JS van den Berg: Second author $-10 \%$

3. Susanna M du Plessis: Third author - $10 \%$
Conclusion

The UPFCD clinic primary surgical protocol displayed a good treatment outcome rating, in line with that of the CCUK cohort and better than the CSAG results.

\section{Keywords}

Cleft in Africa, Cleft audit. cleft outcome, plaster models.

\section{INTRODUCTION}

Despite the advances in the surgical management of patients with facial cleft deformities (FCDs), there are still many controversies regarding the ideal primary surgical protocols for such deformities. ${ }^{1-2}$ Consequently, there is a constant need to assess treatment outcomes of patients treated for cleft lip and palate (CLP) defects to monitor and implement the highest possible standards of care, thus improving treatment protocols for future patients. ${ }^{3}$

Cleft centres in Europe and America have conducted multi-centre studies, for example, Eurocleft and Americleft, both of which resulted in changes being made to the delivery of cleft care. ${ }^{4}$ Although clinical audits have been implemented in different parts of the world, ${ }^{5}$ have not yet been carried out in Pretoria, South Africa. This is a collaborative project established under the guidance of the Dental School in Dundee, Scotland. It is an audit for the current primary surgical treatment outcome of the UPFCD clinic.

\section{LITERATURE REVIEW}

FCDs represent the largest group of craniofacial deformities of the oral structures, with CLP being the most common. ${ }^{6}$ In South Africa, CLP defects incidence is approximately 1.38 per 1000 for the white and 0.42 per 1000 for the black population groups.

Facial growth is one of the key areas of interest for assessing the quality of cleft treatment outcomes. ${ }^{8}$ Ensuring good facial growth may result in dental arch relationships that can be treated conventionally. Surgical correction of the skeletal bases is avoided, thus providing optimal re- 
sults regarding function and facial appearance. It also minimises costs to the patients and health care providers, which consequently lessens the caregiver's burden. ${ }^{4,9}$

The World Health Organization (WHO), in its report "Global strategies to reduce the healthcare burden of craniofacial anomalies," recommended international collaborative research on craniofacial anomalies and issued guidelines for clinical management of patients with CLP. ${ }^{10-11}$

These guidelines have been followed in Europe, North America and other parts of the world. As a result, these regions implemented baseline standards of cleft care with recommendations on improving the quality of cleft services. ${ }^{2}$ In Africa, there are many gaps in the knowledge and assessment of the treatment outcome of CLP care due to the shortage of multidisciplinary cleft care services and cleft specialists. ${ }^{12}$

The UPFCD clinic was established in 1983 and is the largest in Africa. ${ }^{13}$ The clinic is an ideal setting to conduct studies due to the high volume of patients, ethnic diversity, and retrospective records availability. ${ }^{14}$

Treatment outcome studies allow for comparison between different centres and help provide evidence of cleft care success. ${ }^{15}$ These studies also encourage co-operation and collaborative work. ${ }^{3}$ Given the potential impact of primary surgical protocols on dentofacial growth and development, one of the most noteworthy findings of the Eurocleft study was the ability to detect differences in dental arch relationships using a simple, yet popular, outcome measure, namely the Great Ormond Street, London, and Oslo (GOSLON) Yardstick. ${ }^{16}$

The yardstick offers a reliable means of measuring dentofacial relationships, ${ }^{17}$ using a set of dental study models arranged in five groups from the very best dental arch relationships in unilateral CLP subjects (GOSLON Group 1) to the worst (GOSLON Group 5) (Fig. 1).
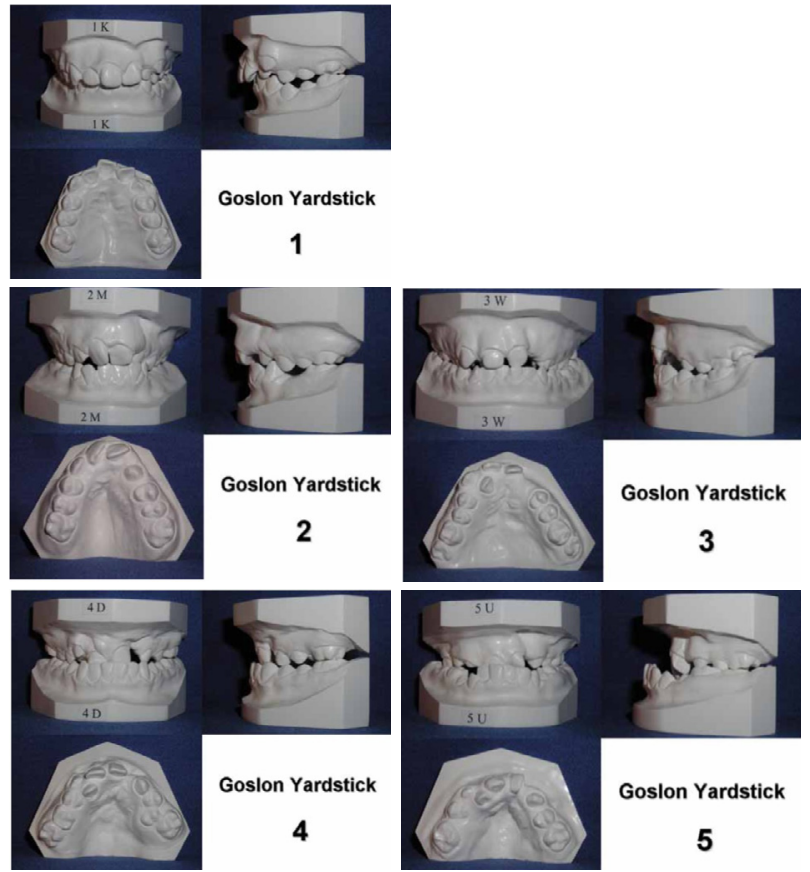

Figure 1. GOSLON Yardstick Index.
Cases falling into Groups 4 and 5 are generally considered so severe that they are beyond orthodontic correction alone and require surgical involvement (Table 1). ${ }^{15}$

\begin{tabular}{|c|c|c|}
\hline Group & Description & $\begin{array}{l}\text { Long-term } \\
\text { outcome }\end{array}$ \\
\hline Group 1 & $\begin{array}{l}\text { Positive overjet with average inclined or re- } \\
\text { troclined incisors with no crossbite or open } \\
\text { bite. }\end{array}$ & Excellent \\
\hline Group 2 & $\begin{array}{l}\text { Positive overjet with average inclined or pro- } \\
\text { clined incisors with unilateral crossbite or } \\
\text { crossbite tendency with or without open } \\
\text { bite tendency around the cleft site. }\end{array}$ & Good \\
\hline Group 3 & $\begin{array}{l}\text { Edge-to-edge bite with average inclined or } \\
\text { proclined incisors or reverse overjet with re- } \\
\text { troclined incisors. Unilateral crossbite with } \\
\text { or without open bite tendency around the } \\
\text { cleft site. }\end{array}$ & Fair \\
\hline Group 4 & $\begin{array}{l}\text { Reverse overjet with average inclined or pro- } \\
\text { clined incisors. Unilateral crossbite with or } \\
\text { without bilateral crossbite tendency with or } \\
\text { without open bite tendency around the cleft } \\
\text { site. }\end{array}$ & Poor \\
\hline Group 5 & $\begin{array}{l}\text { Reverse overjet with proclined incisors, bilat- } \\
\text { eral crossbite, and poor maxillary arch form } \\
\text { and palatal vault anatomy. }\end{array}$ & Very poor \\
\hline
\end{tabular}

Numerous studies have been conducted using this measure. ${ }^{4,16,18}$ They find GOSLON rating is a reliable, rapid, and clinically valid means of assessing dental arch relationships in patients with UCLP defects. The GOSLON rating is used to provide an audit for primary surgical outcomes and thereby identifies procedures that may need to be changed to improve the treatment outcome. ${ }^{19}$ For this reason, the present study was undertaken to follow the worldwide cleft centres' effort to improve CLP care by monitoring the CLP treatment outcome.

\section{OBJECTIVES}

The study objective is to assess the effect of the CLP primary surgery protocol of the UPFCD clinic on the dental arches' relationship, using the GOSLON index rating.

\section{METHODS}

Ethics approval was obtained from the Research Ethics Committee of the University of Pretoria's Faculty of Health Sciences. The sample of 27 consecutively treated patients (average age 12 years with non-syndromic complete UCLP had not received any active orthodontic treatment. All patients in the sample had their primary surgery protocol performed by the same surgeon at the UPFCD clinic.

The plaster study models collected as part of the routine clinical records appointments were duplicated and sent to the Cleft Lip and Palate Centre at the Dental School in Dundee, Scotland. All models were trimmed in the same manner to eliminate bias and ensure that the assessors could not identify from which institution the models were sent. Numbers were randomly assigned to each model and marked in pencil. No other means of identification was recorded. In addition, matching clinical records sourced from the archives and details such as diagnosis, treatment planning, surgical interventions protocol, and treatment outcomes were recorded. 


\section{Data analysis}

The GOSLON index was used for each study model, and it was analysed and rated by a blind panel at the Dental School in Dundee, using standardised rating schemes. Examiners were calibrated and went through repeatability and reliability tests to ensure the consistency of recordings.

\section{Statistical methods}

The Linear Weighted Kappa statistic and Kendall's Coefficient of Concordance statistic were used to determine agreement levels within and between raters.

\section{RESULTS}

There were a strong intra- and inter-rater agreement, with a weighted kappa of 0.92 indicating a very good Kappa values category (Table 2).

\begin{tabular}{|l|l|}
\hline \multicolumn{2}{|l|}{ Table 2. Categorisation of Kappa values. } \\
\hline Value of $\mathbf{K}$ & Strength of agreement \\
\hline$<0.20$ & Poor \\
$0.21-0.40$ & Fair \\
$0.41-0.60$ & Moderate \\
$0.61-0.80$ & Good \\
$0.81-1.00$ & Very good \\
\hline
\end{tabular}

The distributions of the GOSLON score for each centre are shown in Fig. 2.

The mean GOSLON score of the UPCFD clinic was 2.85, which is better than the 3.2 for the CSAG and similar to the CCUK cohort studies of 2.62 (Fig. 3).

\section{DISCUSSION}

\section{Treatment outcome audit}

In agreement with Shaw et al. $^{2}$ and Asher-McDade, Roberts, C. ShawGallager 20 clinical audit and quality assurance to monitor cleft treatment outcome are regarded as a requirement to take remedial action for any treatment shortcomings.

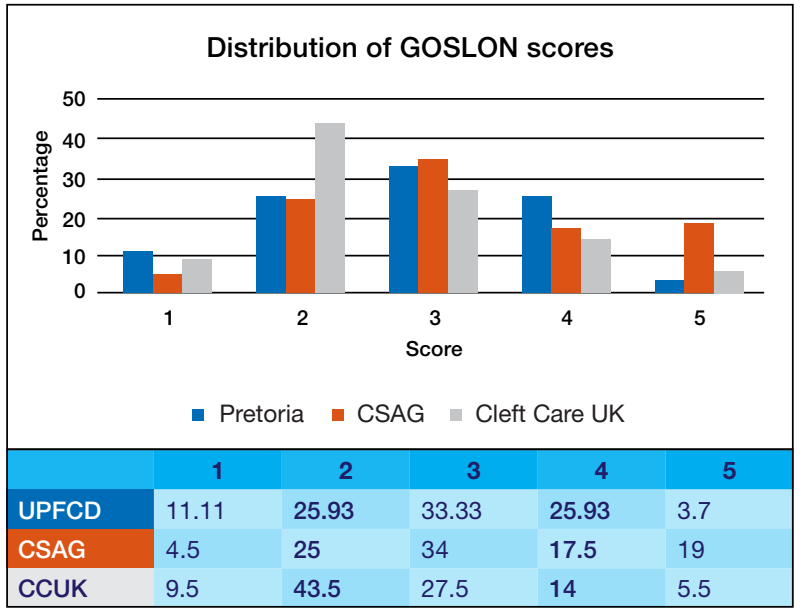

Figure 2. Distribution of GOSLON scores between UPFCD, CSAG, and CCUK.

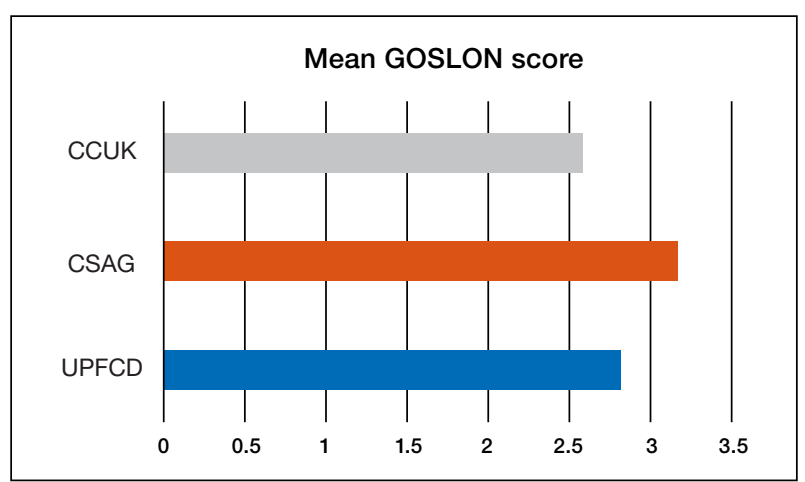

Figure 3. Mean GOSLON scores of UPFCD CSAG and CCUK.

The reason for choosing the GOSLON yardstick index

Assessment of primary cleft surgery's effect on midfacial growth (consequently on the dental arch relationships) is an accepted method..$^{21}$ Different indices have been developed based on scoring the dental arch relationship. ${ }^{22-26}$

From all different indices, the GOSLON yardstick index was chosen to score the sample due to its widespread acceptance and to allow multicentre score comparison. ${ }^{27}$

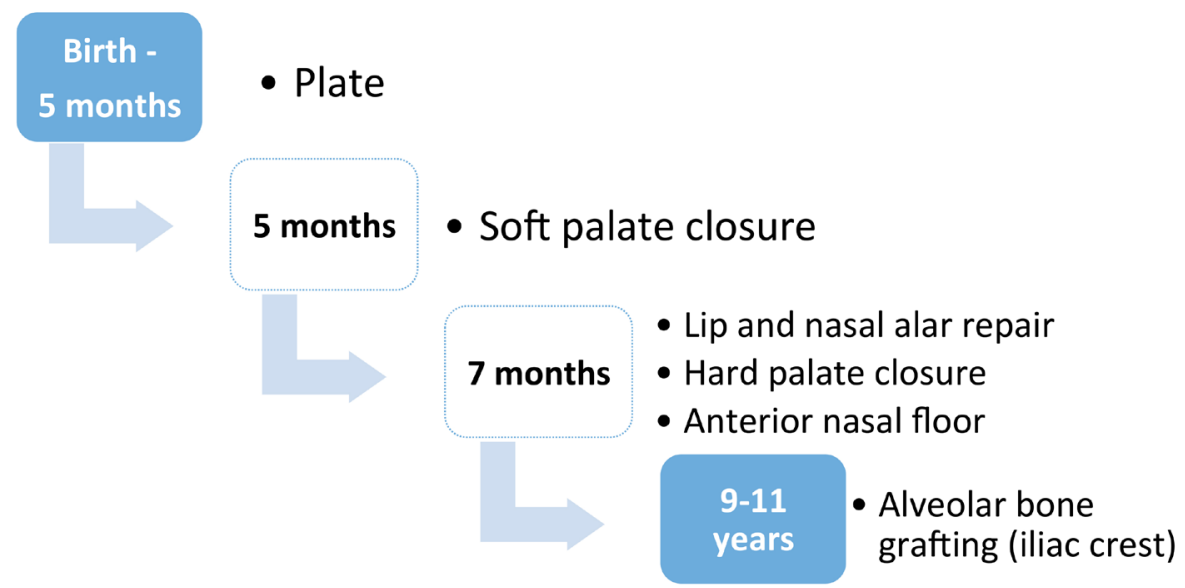




\section{Treatment protocol}

The UCLP care protocol of the UPFCD clinic (Fig. 4) consists of a pre-surgical infant orthopaedic (PSIO) plate inserted at age one to two weeks to assist with feeding and speech development to facilitate primary surgery. ${ }^{28-32}$ At the age of five months, the soft palate is closed and the hard palate at seven months. Alveolar bone grafting is done at ages 9 to 11 years, using harvested bone from the iliac crest.

The UCLP primary surgery protocol includes two-stage palatal closure and delayed lip closure to assist with an intelligible speech before the child articulates and minimises the negative effect on midfacial growth (in agreement with Hollmann, ${ }^{33}$ Precious, Goodday, Morrison, Davis ${ }^{34}$ ). This contrasts with Lilja, Mars, Elander, Enocson, Hagberg, Worrell, ${ }^{35}$ who delayed hard palate closure after three years.

This study found that the hard palate's closure at age seven months did not lower the GOSLON rating. This is in line with the findings of Peterson-Falzone ${ }^{36}$ and Willadsen, Boers, Schöps, Kisling-Møller, Nielsen, Jørgensen. ${ }^{37}$ This study showed that surgery outcomes using different surgical protocols achieved similar GOSLON ratings. ${ }^{38}$

\section{CONCLUSION}

The UPFCD clinic displayed good treatment outcomes comparable to those of the CCUK cohort and the Americleft Study Centre C. This study outcome suggests that the clinical treatment protocol at UPFCD is an effective strategy for treating patients with UCLP regarding favourable maxillary development. Future research is recommended to continue monitoring the UPFCD surgical treatment outcomes and participate in multicentre studies.

\section{Acknowledgments}

The author would like to thank Prof FA de Wet for proofreading the manuscript and the Dundee/UK collaborative team of Dr. G.Mclntyre and Prof P. Mossey for their valuable contributions towards the project. The author would also like to thank Francois du Plessis for his contibution.

\section{References}

1. Long R, Jr., Shaw W, Semb G. Eurocleft and Americleft studies: Experiments in intercenter and international collaboration. In: Berkowitz S, editor. Cleft lip and palate: Springer Berlin Heidelberg. 2013; 929-43.

2. Shaw WC, Williams AC, Sandy JR, Devlin HB. Minimum standards for the management of cleft lip and palate: Efforts to close the audit loop. Royal College of Surgeons of England. Annals of The Royal College of Surgeons of England. 1996; 78(2): 110-4.

3. Shaw WC, Brattstrom V, Molsted K, Prahl-Andersen B, Roberts CT, Semb G. The Eurocleft study: Intercenter study of treatment outcome in patients with complete cleft lip and palate. Part 5: Discussion and conclusions. Cleft Palate Craniofac J. 2005; 42(1): 93-8.

4. Hathaway R, Daskalogiannakis J, Mercado A, Russell K, Long Jr RE, Cohen M, et al. The Americleft study: An intercenter study of treatment outcomes for patients with unilateral cleft lip and palate part 2. Dental arch relationships. The Cleft Palate-Craniofacial Journal. 2011; 48(3): 244-51.
5. Mossey P, Little J. Addressing the challenges of cleft lip and palate research in India. Indian Journal of Plastic Surgery. 2009; 42(S1).

6. Ranalli DN. Design of a postdoctoral training program in the treatment of children with congenital orofacial clefts. Special Care in Dentistry. 1981; 1(5): 218-20.

7. Van Wyk P, Bütow K, van der Merwe C, Kleynhans E. Incidence and clinical appearance of cleft deformities in the Transvaal. The Journal of the Dental Association of South Africa= Die Tydskrif van die Tandheelkundige Vereniging van Suid-Afrika. 1987; 42(7): 403.

8. Shaw WC, Dahl E, Asher-McDade C, Brattstrom V, Mars M, McWilliam J, et al. A six-center international study of treatment outcome in patients with clefts of the lip and palate: Part 5. General discussion and conclusions. Cleft Palate Craniofac J. 1992; 29(5): 413-8.

9. Sischo L, Clouston SA, Phillips C, Broder HL. Caregiver responses to early cleft palate care: A mixed method approach. Health Psychology. 2016; 35(5): 474.

10. Shaw DW. Global strategies to reduce the health care burden of craniofacial anomalies: Report of who meetings on international collaborative research on craniofacial anomalies. The Cleft palate-craniofacial journal. 2004; 41(3): 238-43.

11. WHO. Global strategies to reduce the healthcare burden of craniofacial anomalies: Report of who meetings on international collaborative research on craniofacial anomalies: Geneva, Switzerland, 5-8 November 2000; park city, Utah, USA 24-26 May 2001: Human Genetics Programme, Management of Noncommunicable Diseases, World Health Organization. 2002.

12. Oluwaseun A. Adetayo, Mark C. Martin. Demographics of cleft care providers in Africa and reported experience in training and practice: Direct analysis of continent-based practitioners. The Cleft Palate-Craniofacial Journal. 2012; 49(3): 286-90.

13. Bütow KW. Treatment of cleft lip and palate. Part V: The c linic and the multidisciplinary approach to cleft lip and palate cases. J Dent Assoc S Afr. 1984; 39(8): 543-5.

14. Louw B, Shibambu M, Roemer K. Facilitating cleft palate team participation of culturally diverse families in South Africa. The Cleft palate-craniofacial journal. 2006; 43(1): 47-54.

15. Russell K, Long RE, Jr., Hathaway R, Daskalogiannakis J, Mercado A, Cohen M, et al. The Americleft study: An intercenter study of treatment outcomes for patients with unilateral cleft lip and palate part 5. General discussion and conclusions. Cleft Palate Craniofac J. 2011; 48(3): 265-70.

16. Mars M, Plint DA, Houston WJ, Bergland O, Semb G. The GOSLON yardstick: A new system of assessing dental arch relationships in children with unilateral clefts of the lip and palate. Cleft Palate J. 1987; 24(4): 314-22.

17. Harila V, Ylikontiola LP, Sandor GK. Dental arch relationships assessed by GOSLON yardstick in children with clefts in northern Finland. Eur J Paediatr Dent. 2014; 15(4): 389-91.

18. Mars M, Houston WJ. A preliminary study of facial growth and morphology in unoperated male unilateral cleft lip and palate subjects over 13 years of age. The Cleft PalateCraniofacial Journal. 1990; 27(1): 7-10.

19. Fudalej P, Hortis-Dzierzbicka M, Obloj B, Miller-Drabikowska D, Dudkiewicz Z, Romanowska A. Treatment outcome after one-stage repair in children with complete unilateral cleft lip and palate assessed with the GOSLON yardstick. Cleft Palate Craniofac J. 2009; 46(4):374-80.

20. Asher-McDade C, Roberts C, Shaw W, Gallager C. Development of a method for rating nasolabial appearance in patients with clefts of the lip and palate. The Cleft PalateCraniofacial Journal. 1991; 28(4): 385-91.

21. Daskalogiannakis J, Mercado A, Russell K, Hathaway R, Dugas G, Long Jr RE, et al. The Americleft study: An intercenter study of treatment outcomes for patients with unilateral cleft lip and palate part 3. Analysis of craniofacial form. The Cleft Palate-Craniofacial Journal. 2011; 48(3): 252-8. 
22. Shaw WC, Richmond S, O'Brien KD. The use of occlusal indices: A European perspective. Am J Orthod Dentofacial Orthop. 1995; 107(1): 1-10.

23. Mossey P, Clark J, Gray D. Preliminary investigation of a modified HUDDART/BODENHAM scoring system for assessment of maxillary arch constriction in unilateral cleft lip and palate subjects. The European Journal of Orthodontics. 2003; 25(3): 251-7.

24. Pruzansky S, Aduss H. Arch form and the deciduous occlusion in complete unilateral clefts. The Cleft palate journal. 1964; 1(4): 411-8.

25. Huddart A, Bodenham R. The evaluation of arch form and occlusion in unilateral cleft palate subjects. The Cleft palate journal. 1972; 9(3): 194-209.

26. Dobbyn L, Gillgrass T, Mclntyre G, Macfarlane T, Mossey P. Validating the clinical use of the modified HUDDART and BODENHAM scoring system for outcome in cleft lip and/ or palate. The Cleft palate-craniofacial journal. 2015; 52(6): 671-5.

27. Long Jr RE, Hathaway R, Daskalogiannakis J, Mercado A, Russell K, Cohen M, et al. The Americleft study: An inter-center study of treatment outcomes for patients with unilateral cleft lip and palate part 1. Principles and study design. The Cleft Palate-Craniofacial Journal. 2011; 48(3): 239-43.

28. Papadopoulos MA, Koumpridou EN, Vakalis ML, Papageorgiou SN. Effectiveness of pre-surgical infant orthopedic treatment for cleft lip and palate patients: A systematic review and meta-analysis. Orthodontics \& Craniofacial Research. 2012; 15(4): 207-36.

29. Larson M, Sällström KO, Larson O, McWilliam J, Ideberg M. Morphologic effect of preoperative maxillofacial orthopedics (t-traction) on the maxilla in unilateral cleft lip and palate patients. The Cleft palate-craniofacial journal: official publication of the American Cleft Palate-Craniofacial Association. 1993; 30(1): 29-34.

30. Hotz MM. Pre-and early postoperative growth-guidance in cleft lip and palate cases by maxillary orthopedics (an alternative procedure to primary bone-grafting). The Cleft palate journal. 1969; 6(4): 368-72.

31. Koželj VDDSPD. Changes produced by pre-surgical orthopedic treatment before cheiloplasty in cleft lip and palate patients. The Cleft Palate-Craniofacial Journal. 1999; 36(6): 515-21.

32. Bruce Ross R. Treatment variables affecting facial growth in unilateral cleft lip and palate. Part 2: Presurgical orthopaedics. 1986.

33. Hollmann K. "Vienna concept" of treatment of cleft lip, jaw and/or palate. Osterreichische Zeitschrift fur Stomatologie. 1980; 77(7-8): 249.

34. Precious DS, Goodday RH, Morrison AD, Davis BR. Cleft lip and palate: A review for dentists. Journal-Canadian Dental Association. 2001; 67(11): 668-73.

35. Lilja J, Mars M, Elander A, Enocson L, Hagberg C, Worrell E, et al. Analysis of dental arch relationships in Swedish unilateral cleft lip and palate subjects: 20-year longitudinal consecutive series treated with delayed hard palate closure. The Cleft palate-craniofacial journal. 2006; 43(5): 606 -11.

36. Peterson-Falzone SJ. Optimal age for palatoplasty to facilitate normal speech development: What is the evidence? Cleft lip and palate: Springer; 2013: 787-802.

37. Willadsen E, Boers M, Schöps A, Kisling-Møller M, Nielsen JB, Jørgensen LD, et al. Influence of timing of delayed hard palate closure on articulation skills in 3-year-old danish children with unilateral cleft lip and palate. International journal of language \& communication disorders. 2018; 53(1): 130-43.

38. Nollet PJ, Katsaros C, Van't Hof MA, Kuijpers-Jagtman AM. Treatment outcome in unilateral cleft lip and palate evaluated with the GOSLON yardstick: A meta-analysis of 1236 patients. Plast Reconstr Surg. 2005; 116(5): 1255-62. 